\title{
Ionosperic anomaly due to seismic activities - Part 1: Calibration of the VLF signal of VTX 18.2 KHz station from Kolkata and deviation during seismic events
}

\author{
S. Sasmal ${ }^{1}$ and S. K. Chakrabarti ${ }^{1,2}$ \\ ${ }^{1}$ Indian Centre for Space Physics, 43 Chalantika, Garia Sation Road, Kolkata 700084, India \\ ${ }^{2}$ S. N. Bose National Centre for Basic Sciences, JD Block, Salt Lake, Kolkata 700098, India
}

Received: 27 June 2009 - Accepted: 5 August 2009 - Published: 11 August 2009

\begin{abstract}
VLF signals are long thought to give away important information about the lithosphere-ionosphere coupling. In order to establish co-relations, if any, between the ionospheric activities and the earthquakes, we need to understand what the reference signals are, throughout the year. The best opportunity to do this is during the period of solar minimum where the number of flares and sunspots are negligible and the data would be primarily affected by the sun and variation would be due to normal sunset and sunrise effects. In this paper, we present the result of the sunrise and sunset terminators as a function of the day of the year for a period of four years, viz, 2005-2008 when the solar activity was very low. The terminators are for the $18.2 \mathrm{KHz}$ VTX signal of the Indian Navy as observed from Indian Centre for Space Physics receiving station located in Kolkata. A total of 624 days of data have been used to obtain the mean plot. Any deviation of observations from this so-called the standardized calibration curve would point to influences by terrestrial (such as earthquakes) and extra-terrestrial events (such as solar activities). We present examples of deviations which occur in a period of 16 months and show that the correlation with seismic events is significant and typically the highest deviation takes place up to a couple of days prior to the seismic event. Simultaneous observations of such deviations from more than one station could improve the predictability of earthquakes.
\end{abstract}

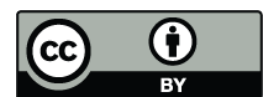

Correspondence to: S. K. Chakrabarti (chakraba@bose.res.in)

\section{Introduction}

It has been reported in the literature that the sun-rise and sun-set terminators (hereafter SRT and SST respectively) could be shifted towards nighttime just before earthquakes (e.g., Gokhberg et al., 1989; Gufeld et al., 1992, Molchanov et al., 1998; Molchanov and Hayakawa, 1998; Clilverd, Rodger and Thomson, 1999; Hayakawa and Molchanov, 2000; Hayakawa et al., 2003; Chakrabarti et al., 2005; Maekawa et al., 2006; Rozhnoi et al., 2009). Similarly, it has been reported that the time taken to form the D-region or to dissolve it through recombination also becomes anomalous immediately prior to the earthquake (Chakrabarti et al., 2007). Though the relationship between terminators and seismicity is clearly not understood well (e.g., Rodger, Clilverd and Thomson, 1999), in order to establish a consensus in this issue, especially in the context of Indian scenario, Indian Centre for Space Physics (ICSP) has been recording the VLF signal for the last several years transmitted from the Indian Navy station VTX near the southern tip of India. Since the signal is affected very much by the solar flares and other terrestrial events, we were interested to acquire data during the solar minimum so that the SRTs and SSTs are as "clean" as possible. The curve which is the locus of all the mean terminator times obtained each day would then be used as a "standardized calibration curve" (SCC) for the VTX-ICSP baseline. Clearly, such a calibration curve not only depends on baseline between a transmitter and a receiver, but when the transmitter emits a multitude of signals, the curve should also depend on the transmitting frequency. At ICSP we are involved in preparing such SCCs for a large number of baselines in order that the ionospheric anomalies may be pinpointed very accurately.

Published by Copernicus Publications on behalf of the European Geosciences Union. 


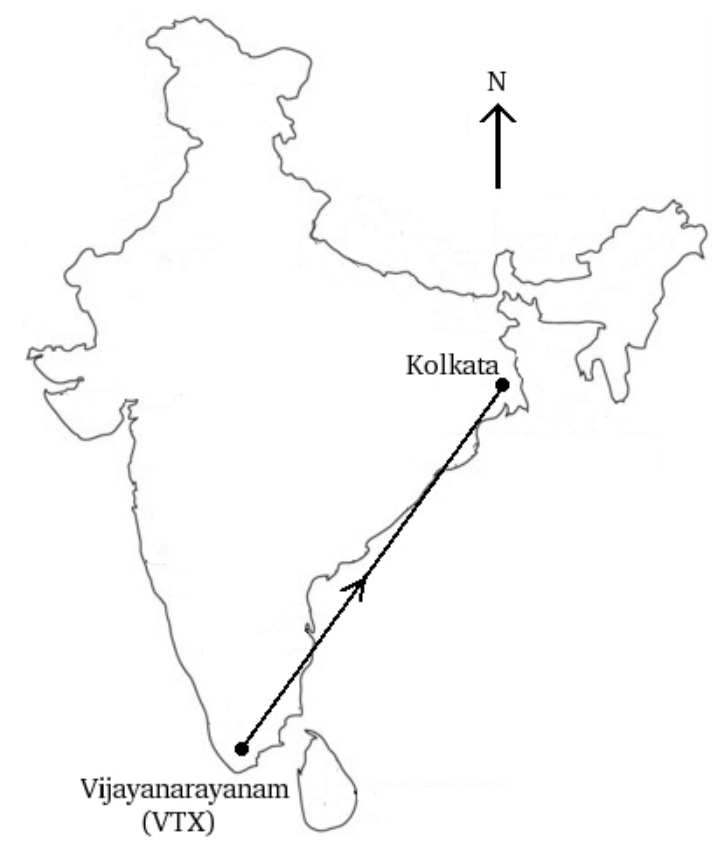

Fig. 1. The West to East great circle path from VTX to Kolkata.

In this first paper of the series, which deals with our efforts to understand the correlation between the seismic and ionospheric activities, we present the so-called SCCs for the VTX-ICSP baseline. We present 624 days of data taken over four years of solar minimum period 2005-2008 and plot their mean behaviour. Several papers are present in the literature to study such long term effects in other parts of the world (e.g., Clilverd, Thomson and Rodger, 1999; Thomson and Clilverd, 2000). We note that the terminator times fall within the local sunrise and sunset times of the transmitter and the receiver. We generally find that the D-layer preparation time (DLPT) is of the same order as the difference in sunrise times (DTSR) between ICSP and VTX in Summer but several times higher in winter. The reverse is true for the D-layer disappearance time (DTDT) when compared to the difference in sunset times (DTSS) between ICSP and VTX. We find that there are several days with an anomalous behavior in the SST and SRT. We choose our data for a period of 16 months and separated those which correspond to significant deviations from our standard calibration curve. We find that a significant correlation exists between these special days with Earthquake events. However, to have a predictibility, we must have similar calibrations with several other stations and find cross-correlations.

The plan of the present paper is the following: In the next section we present our setup. In Sect. 3, we present typical VTX $18.2 \mathrm{KHz}$ signals as received from Kolkata. In Sect. 4, we present results for four years and present a mean calibration curve. We also compare the DLPT/DLDT with DSRT/DSSTs for a given year. In Sect. 5, we present ex- amples of correlations between significant seismic activities and anomalous "VLF days". Finally, in Sect. 6 we draw our conclusions.

\section{The transmitter and the receivers}

Indian Centre for Space Physics (ICSP) is located in Kolkata (Lat. $22^{\circ} 34^{\prime}$, Long. $88^{\circ} 24^{\prime}$ ) and receives signals from several stations throughout the globe. The Indian Navy's VLF transmitter station VTX is located at Vijayanarayanam (Lat. $08^{\circ} 26^{\prime}$, Long. $77^{\circ} 44^{\prime}$ ) near the southern tip of the Indian sub-continent from where all the three oceans, namely, Bay of Bengal, Indian Ocean and Arabian Sea are almost equidistant. Though VTX is known to transmit in at least four frequencies $(16.3 \mathrm{KHz}, 17.0 \mathrm{KHz}, 18.2 \mathrm{KHz}$ and $19.2 \mathrm{KHz}$ ) in this paper, we will present the data received from the VTX signal at $18.2 \mathrm{KHz}$. At VTX there are altogether 13 antennas placed on a six cornered star configurations with antennas places about a $\mathrm{km}$ from each other. Figure 1 shows the West-East path from VTX to Kolkata. The distance between VTX and ICSP is $1941 \mathrm{~km}$. We primarily use the AWESOME receiver of Stanford University (http: //www-star.stanford.edu/ vlf/) with two cross-loops which are capable of receiving fluctuating magnetic fields in the VLF signals. Both broadband and narrow-band data are obtained from both the East-West and North-South antennas. The phase information was also obtained from each antenna. The data is automatically stored in the computer after it is pre-amplified and time-stamped by GPS a unit. As a back up, we also use our Gyrator-II type receiver capable of tuning in to a single transmitter. The latter antenna is a single loop type. In some days, the terminator times from the loop antenna was used for the calibration.

\section{Typical VLF Signals from VTX}

In Fig. 2a-b we present the amplitude of VTX signals as a function of time. Here we chose the Indian Standard Time (IST=UT+5:30:00) to plot the data. We chose two cases. In Fig. 2a, the data of 19 January 2007 is presented. The signal clearly showed recovery after the sunrise terminator and before the sunset terminator. The "cosine" pattern of the solar flux is clearly visible in the daytime. The formation of D-layer in the morning is marked by sharp attenuation of the signal from about 120 to about 20. The disappearance of the D-layer in the evening is seen by the sharp enhancement in the signal. In Fig. 2b, the data of 6 May 2007 is presented, where we note that the morning and evening "recovery" bumps in the signal are completely absent. For the sake of future purpose, we marked the sun-rise terminator (SRT) and sun-set terminator (SST). We define SRT by the first weak minimum which occur after the weakening of the signal in the morning. We define SST by last weakening of the signal before the complete disappearance of the D-layer. 

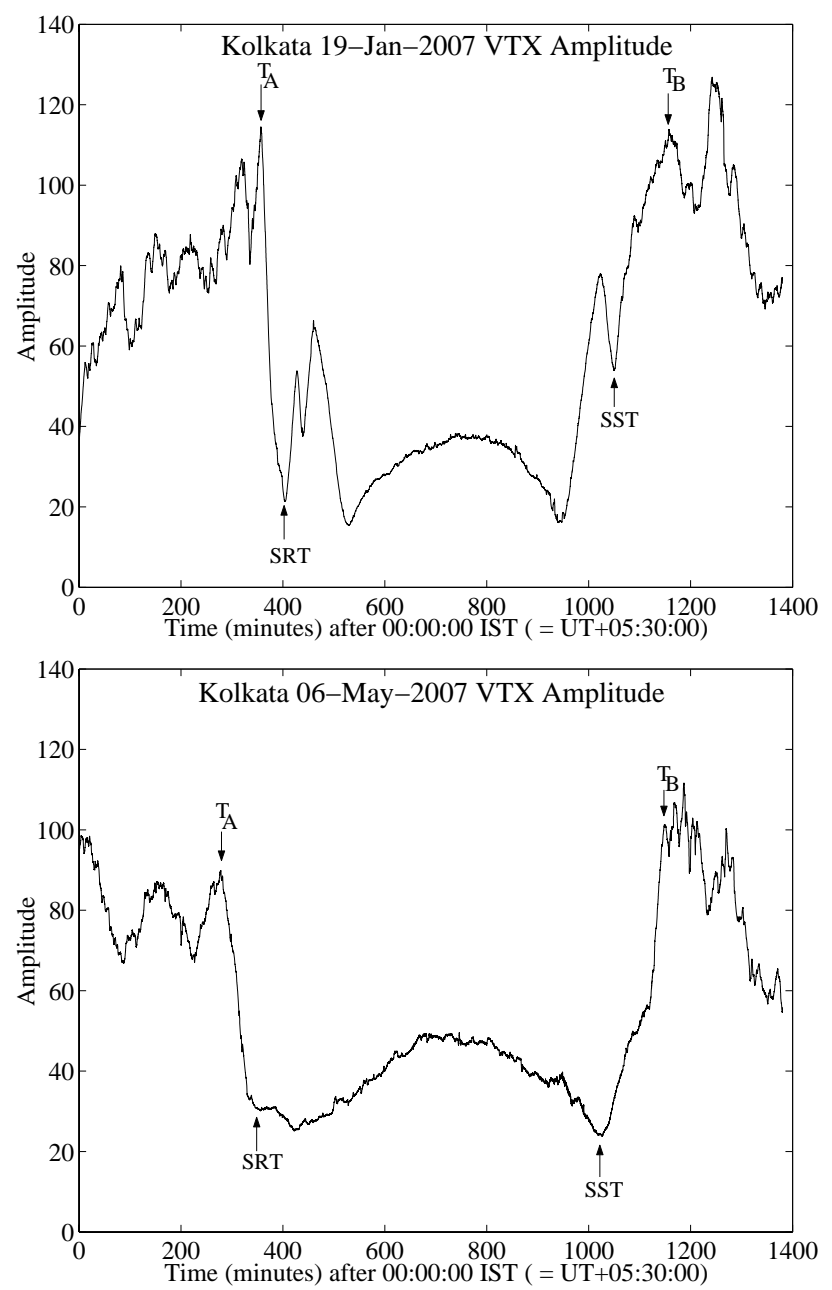

Fig. 2. The variation of the amplitude of the VTX signal as a function of time in minutes on two specific days: (a) 19 January 2007 and (b) 6 May 2007. The locations of the sunrise terminator (SRT) and sunset terminator (SST) used by the rest of the paper are marked. In (a), the signal clearly showed recovery after the sunrise terminator and before the sunset terminator but in (b) these patterns are absent. The "cosine" pattern of the solar flux is clearly visible in the daytime. We place $T_{A}$ to denote the time from where the signal starts to attenuate towards SRT and $T_{B}$ to denote the time when the signal is fully recovered after the sunset terminator.

Thus in Fig. 2b, the only minimum occurring in the evening is considered as SST. In both the plots, we place $T_{A}$ to denote the time from where the signal starts to attenuate towards sunrise terminator in the morning and $T_{B}$ to denote the time when the signal is fully recovered after sunset terminator (marked by arrows). Thus, SRT- $T_{A}$ could notionally be called D-layer preparation time or DLPT and $T_{B}$-SST could notionally be called D-layer disappearance time or DLDT.

In order to see the quality of the VTX signal throughout the year, in Fig. 3, we present the data taken on 21 st of every

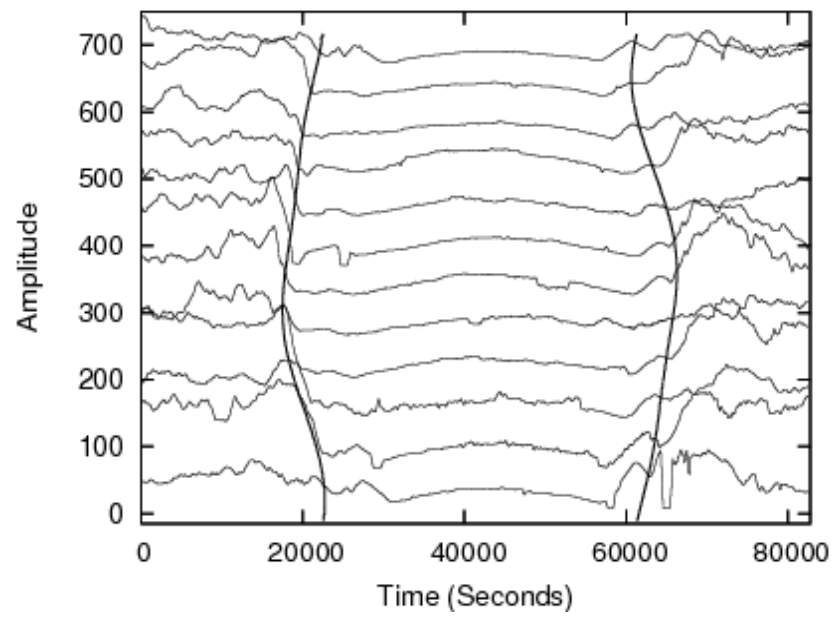

Fig. 3. The data of 21 st of every month for the year 2007 is stacked after an amplitude shift of 60 units for each month for clarity. The thick solid curves are the sunrise and sunset times at Kolkata.

month for the year 2007, that of January being at the bottom. The amplitude is shifted by 60 units for each month in order that they may be distinguished. The thick solid curves are the sunrise and sunset times at Kolkata. They were plotted just to guide the eyes their relationship with the sunrise and sunset terminators in the signal.

\section{Standardized Calibration Curves (SCCs)}

In order to obtain the "mean" SRT and SSTs over the year we now present four years of data (2005-2008). We removed those SRTs and SSTs from the plot in which there was evidence for solar activity. We have not removed the data for those days in which the SRT or SST may be anomalous due to seismic activities, for example. This is because we did not have any reference frame to quantify the disturbance. However, once the mean SCCs are drawn for the sunrise and sunset, significant deviations from the mean may be used to judge whether there is any real correlation between the ionospheric and lithospheric activities.

In Fig. 4 we plot the SRTs and SSTs by circles. The solid marked curves are for sunrise time at Kolkata (SRKOL), sunrise time at VTX station (SR-VTX), sun-set time at Kolkata (SS-KOL) and sun-set time at VTX (SS-VTX). The lengths of the day at Kolkata [TD(KOL)] and VTX [TD(VTX)], obtained from the time difference on each day are also plotted. Circles mark the sunrise and sunset terminator times (SRTs and SSTs) obtained from our data. We note that generally SRTs and SSTs fall in between the local sunrise and sunset times, though in some days there are exceptions. The days with exceptions may be related to anomalous ionospheric conditions, and the correlations with possible seismic events will be discussed elsewhere. It is generally 


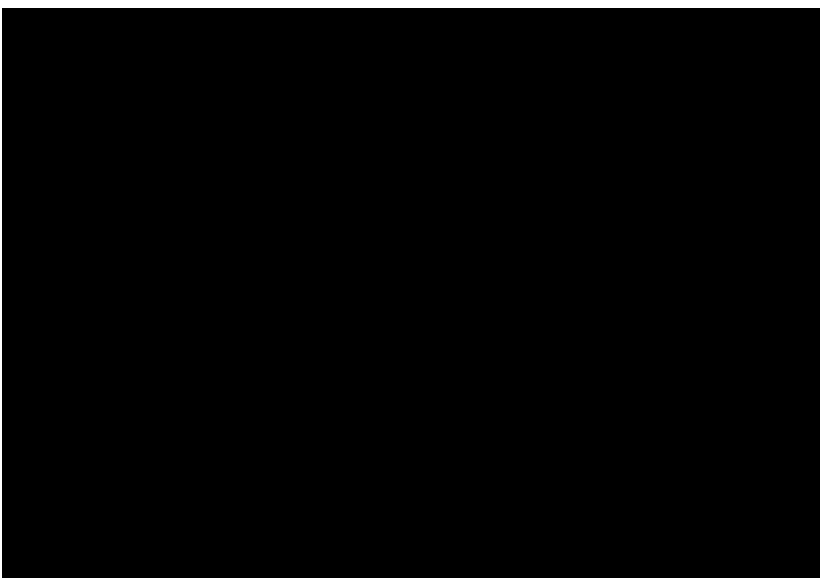

Fig. 4. Comparison of the SRTs and SSTs with the local sunrise and sunset times at Kolkata and VTX. The solid curves are for sunrise time at Kolkata (SR-KOL), sunrise time at VTX station (SR-VTX), sun-set time at Kolkata (SS-KOL) and sun-set time at VTX (SSVTX). Open circles are the terminator times obtained from our data. The lengths of the day at Kolkata [TD(KOL)] and VTX [TD(VTX)], obtained from the time difference on each day are also plotted. Circles mark the sunrise and sunset terminators (SRTs and SSTs) obtained from our data.

observed that DT=SST-SRT, the time between the terminators, in the winter period is closer to $\mathrm{TD}(\mathrm{KOL})$, the length of the day in Kolkata. However, in summer, DT is somewhat intermediate between TD(KOL) and TD(VTX).

In Fig. 5 we superpose all the four years' data into a single plot to obtain the standardized calibration curve (SCC) for the VTX-Kolkata baseline. There are some gaps in the data especially in mid-March and mid-September each year. They are generally due to maintenance of the transmitter or receiving systems. The open circles, closed circles, upper triangles and lower triangles are for the years 2005, 2006, 2007, and 2008 respectively. For each day, the mean is obtained from all the years' data, although it is not necessary that every day we have four points. Thus we did not provide any error bar on the mean curve. Thin solid curves are for sunrise and sunsets in Kolkata (SR-KOL and SS-KOL) and the dashed curves are for sunrise and sunsets at VTX station (SR-VTX and SS-VTX). The thick curves, which are our standardized calibration curve for the VTX-KOL baseline, are obtained by taking running means of all the observed points. In future, these curves will be used to quantify anomalous ionospheric behaviors. Note that the SCC curve is situated roughly in the middle of the SR and SS curves. This shows that the terminators are dominated by a single hop and the location where the signal is reflected is halfway between the transmitter and the receiver.

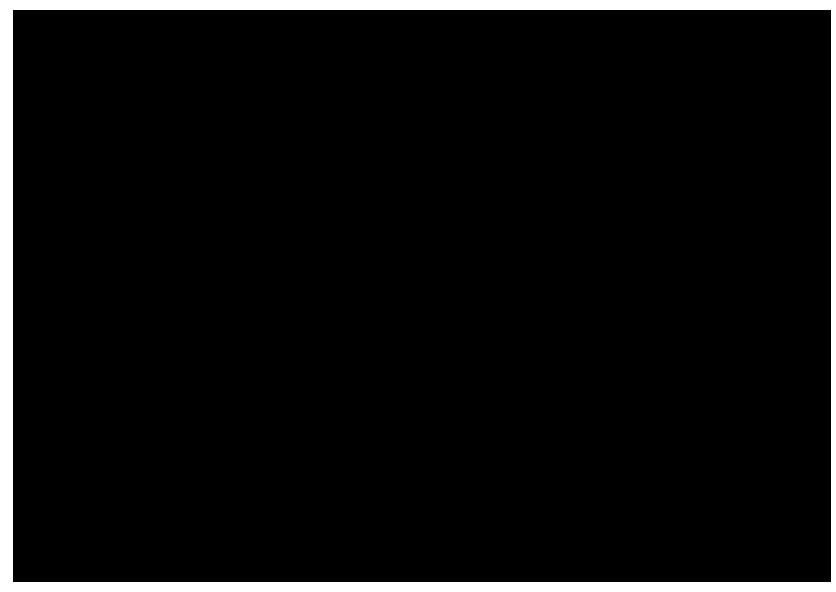

Fig. 5. Superposition of four years of data into a single plot to obtain the standardized calibration curve (SCC). The open circles, closed circles, upper triangles and lower triangles are for the years 2005 , 2006, 2007, and 2008 respectively. Thin solid curves are for sunrise and sunsets in Kolkata (SR-KOL and SS-KOL) and the dashed curves are for sunrise and sunsets at VTX station (SR-VTX and SS-VTX). The thick curves, which are our standardized calibration curve for the VTX-KOL baseline, are obtained by taking running means of all the observed points.

\section{The correlation with seismic activities}

It has been variously suggested in the literature (see, Sect. 1) that there could be some correlation between the length of the "VLF day", i.e., the time taken between the terminators, and the seismic activities. To see if such correlations exist, we use the data for the period November 2006 to February 2008. First, we plot the day-length as a function of day in Fig. 6 by filled circles. To obtain the mean variation (dashed curve) during the period, we took the running mean of only the "inactive" days, i.e., the days where the SRT and SRT are within $2 \times \sigma_{\text {whole }}$ of the whole data. From the data of the inactive days defined in this way, we compute the standard deviation $\sigma$ and ploted curves which are $\sigma, 2 \sigma$ and $3 \sigma$ away from the mean (solid, dotted and dash-dotted respectively). In some days anomalous deviations, as high as $5 \sigma$ levels, are seen. Some seismic activities were generally found to occur in those or nearby days. We associated such activities with those anomalous days and marked the association by larger gray circles. These circles are kept at a constant distance away from the observed point (filled circle), and thus, their exact co-ordinates are not important.

To find out the correlation with the seismic activity we take the seismic events which occurred in the neighborhood of great circle path between the tramsmitter and the receiver. We compile the latitude and longitude of the places of the earthquakes, its magnitude, the depth of the epicenter from the Indian Meteorological Department (http://www.imd.ernet.in). The actual magnitudes of the 


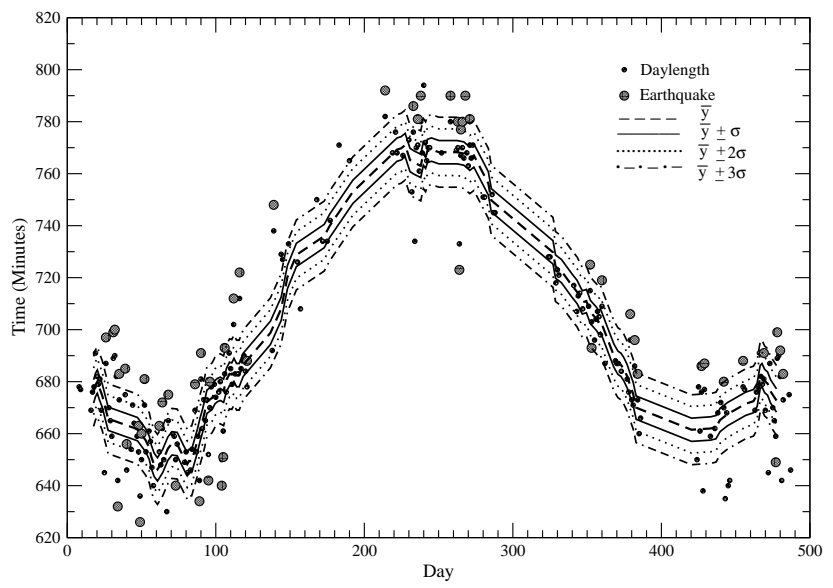

Fig. 6. Variation of the mean "VLF-days", i.e., length of time between the sunrise and sunset terminators, (dashed curve) from a period of 4 November 2006 to February, 2008. The $\pm 1 \sigma, \pm 2 \sigma, \pm 3 \sigma$ curves are also drawn (solid, dotted and dash-dotted respectively). These are drawn from data of the "normal days", i.e., data which are within the mean by $2 \sigma_{\text {whole }}$. The small filled circles represent all the observed data. The dashed larger circles representing seismic activities are placed on days at a constant distance from the filled circles) just to represent which observation they are thought to be associated with, even though the actual seismic event might have taken place after that day.

earthquakes are of no concern since we are interested in the energy deposition by these earthquakes near the mid-point of the great circle path Instead of taking the original magnitude, we calculate the energy of these earthquakes from: $\log _{10} E=4.4+1.5 M_{s}$, for earthquake less than 5.0 magnitude, and $\log _{10} E=5.24+1.44 M_{s}$, for earthquake greater than 5.0 magnitude, where, $E=$ Energy of the earthquake in Jules, and $M_{s}$ =surface wave magnitude (Lowrie, 2007).

In the days when more than one earthquake occurs, we calculate the total energy from the individual earthquakes and take an average. From this, by reverse process, we find out the effective magnitude on each day. We thus have the deviation of the VLF-daylength from the mean and the effective magnitude on any given day (effective magnitude is zero on non-seismic days) which enable us to compute the correlation coefficients. Figure 7 shows the correlation of these two quantities. We observe that the correlation peaks at two days ahead of the earthquake. Thus it is possible that some ionospheric anomaly occurs prior to the earthquake.

\section{Conclusions}

In this paper, we introduced the concept of having a Standardized Calibration Curve or SCC for a baseline defined as the Transmitter to Receiver great circle path for the entire period of the year. For this purpose we have used the four years

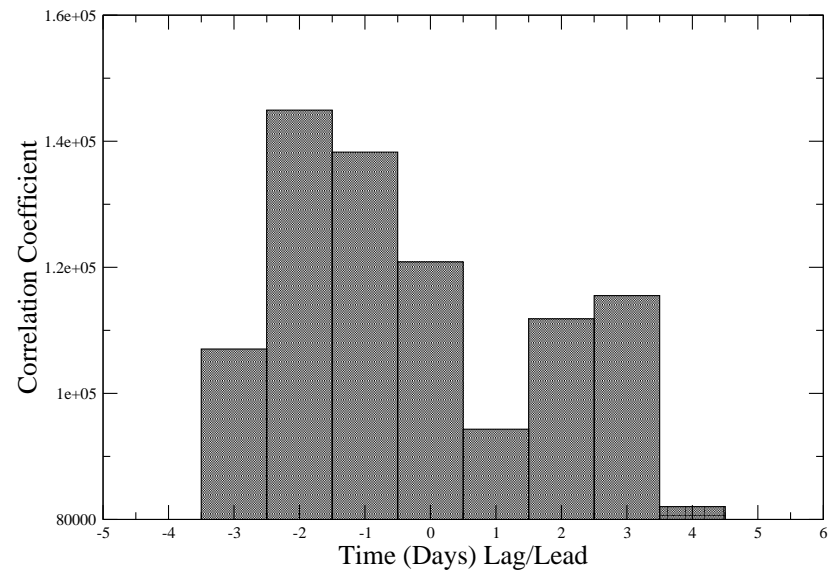

Fig. 7. Correlation between the effective magnitude of the earthquake at the mid-point between the transmitter and the receiver, and the deviation of VLF-day length. Note that the correlation peaks 2 days before the earthquake and is generally higher before and not after the seismic event.

of data from 2005 to 2008 when there was very low solar activity. The mean curve that we draw through the sunrise and sunset terminator times can be used in a number of ways, particularly to quantify any deviation arising out of solar, extraterrestrial and terrestrial activities. Since solar flares could be eliminated from satellite data, we can have opportunities to correlate the deviations with probable ionizing effects of seismic events.

In order to check if deviations occurred due to seismic events, we took sixteen months of seismic data and found correlations between the effective magnitudes of the earthquakes with the deviation from the mean. The correlation shows that in majority of the cases deviation may occur two days before the seismic event. Though the unusual sunrise/sunset terminator times may have some relation with seismicity, it was found to be difficult to predict the location of the seismic centers just on the basis of the data from one station only. For this, we require that the SCCs are to be obtained for several transmitter-receiver baselines continuously. If such deviations are observed to a greater extent along a certain baseline and to lesser extents along the others, one could imagine that seimicity is expected along the former.

There is another way to quantify the ionospheric activities. In the text, we have already introduced DLPT and DLDT. In Chakrabarti et al. (2007) a preliminary discussion was made to show that these quantities may also be used to study ionospheric anomalies. In our next paper, (Chakrabarti, Sasmal and Chakrabarti, 2009) we will show the correlations of DLPT and DLDT with the seismic activities. 
Acknowledgements. We acknowledge many helpful discussions with S. Chakrabarti, Morris Cohen and R. Bhattacharyya. This project was supported by a grant from RESPOND of ISRO.

Edited by: M. E. Contadakis

Reviewed by: two anonymous referees

\section{References}

Chakrabarti, S. K., Saha, M., Khan, R., Mandal, S., Acharyya, K., and Saha, R.: Possible Detection of Ionospheric Disturbances during Sumatra-Andaman Islands Earthquakes in December 2004, Indian J. Radio and Space Phys., 34, 314-317, 2005.

Chakrabarti, S., Sasmal, S., Saha, M., Khan, R., Bhowmik, D., and Chakrabarti, S. K.: Unusual behaviour of D-region Ionization time at $18.2 \mathrm{KHz}$ during seismically active days, Indian J. Phys., 81(5 and 6), 531-538, 2007.

Chakrabarti, S. K., Sasmal, S., and Chakrabarti, S.: Ionosperic anomaly due to seismic activities - Part 2: Possible evidence from D-layer preparation and disappearance times, Nat. Hazards Earth Syst. Sci., in preparation, 2009.

Clilverd, M. A., Rodger, C. J., and Thomson, N. R.: Investigating seismoionospheric effects on a long subionospheric path, J. Geophys. Res., 104(A12), 28171-28179, 1999.

Clilverd, M. A., Rodger, C. J., and Thomson, N. R.: Sunrise effects on VLF signals propagating over a long north-south path, Rad. Sci., 34(4), 939-948, 1999.

Gokhberg, M. B., Gufeld, I. L., Rozhnoy, A. A., Marenko, V. F., Yampolsky, V. S., and Ponomarev, E. A.: Study of seismic influence on the ionosphere by super long-wave probing of the Earth-ionosphere waveguide, Phys. Earth Planet. Inter. 57, 6467, 1989.
Gufel'd, I. L., Roznoy, A. A., Tyumentsev, S. N., Sherstyuk, S. V., and Yampol'skiy, V. S.: Radio wave field disturbances prior to Rudbar and Rachinsk earthquakes, Physics of the Solid Earth, 3, 267-270, 1992.

Hayakawa, M., Molchanov, O. A., Shima, N., Shvets, A. V., and Yamamoto, N.: "eismo Electromagnetics: Lithosphere Atmosphere-Ionosphere Couplings, edited by: Hayakawa, M. and Molchanov, O. A., TERRAPUB, Tokyo, Japan, p. 223, 2003.

Lowrie, W.: Fundamentals of Geophysics, Cambridge University press, 2007.

Maekawa, S., Horie, T., Yamanuchi, T., Sawaya, T., Ishikawa, M., Hayakawa, M., and Sasaki, H.: A. Statistical study on the effect of earthquakes on the ionosphere, based on the sub-ionospheric LF propagation data in Japan, Ann. Geophys., 24, 2219-2225, 2006, http://www.ann-geophys.net/24/2219/2006/.

Molchanov, O. A. and Hayakawa, M.: Subionospheric VLF signal perturbations possibly related to earthquakes, J. Geophys. Res., 103, 17489-17510, 1998.

Molchanov, O. A., Hayakawa, M., Ondoh, T., and Kawai, E.:Precursory effects in the subionospheric VLF Signals for the Kobe earthquake, Phys. Earth planet. Inter. 105, 239-248, 1998.

Rodger, C. J., Clilverd, M. A., and Thomson, N. R.: Modeling of subionospheric VLF signal perturbations associated with earthquakes, Rad. Sci., 34(5), 1177-1185, 1999.

Rozhnoi, A., Solovieva, M., Molchanov, O., Schwingenschuh, K., Boudjada, M., Biagi, P. F., Maggipinto, T., and Castellana, L.: VLF signal precursors of L'Aquila earthquake, EGS General Assembly, 2009.

Thomson, N. R. and Clilverd, M. A.: Solar cycle changes in daytime VLF sub-ionospheric attenuation, J. Atmos. Sol-Terr. Phys, 601-608, 2000. 